\title{
3
}

\section{RESONANCE ENERGY TRANSFER}

\author{
David L. Andrews, ${ }^{1}$ David S. Bradshaw, ${ }^{1}$ \\ RAYOMOND Dinshaw, ${ }^{2}$ AND GREGory D. SCHOLES ${ }^{2}$ \\ ${ }^{I}$ School of Chemistry, University of East Anglia, Norwich Research Park, Norwich, \\ Norfolk, UK \\ ${ }^{2}$ Department of Chemistry, Institute for Optical Sciences and Centre for Quantum \\ Information and Quantum Control, University of Toronto, Toronto, ON, Canada
}

\subsection{INTRODUCTION}

The absorption of ultraviolet or visible light by an atom or molecule, resulting in its electronic excitation, is a familiar and staple photophysical process. Owing to the quantization of electronic states, the associated ultraviolet/visible absorption spectrum contains bands reflecting fixed energy gaps. It is of significant interest to ascertain the destination of the discrete energy held within an electronic excitation, and the mechanism by which the excitation arrives there. One such photodynamical process involves energy transfer from one molecule (or atom) to another. Remarkably, this "hopping" occurs over spatial dimensions that are typically much larger than the cross-section of a molecule and, in some cases, the effect is completed on a timescale in the sub-picosecond range. This chapter gives an introduction to the key aspects of this energy transfer, from its photophysics to its applications to molecular biology.

Formally, resonance energy transfer (RET) is a photophysical process wherein excitation is transferred from an initially populated donor molecule (or chromophore) to an acceptor molecule through intermolecular interactions. It is a ubiquitous process found in numerous systems including conjugated polymers, light-harvesting proteins, and various other multichromophore systems. A common application of energy transfer-and, indeed, one deployed in nature for solar energy conversion

Photonics: Scientific Foundations, Technology and Applications, Volume IV, First Edition. Edited by David L. Andrews.

(C) 2015 John Wiley \& Sons, Inc. Published 2015 by John Wiley \& Sons, Inc.
Au: Author names "David L. Andrews", "David S. Bradshaw", "Gregory D. Scholes", and Affiliation 1 seem to be mismatch with that of list of contributors. Please check and confirm which one should be followed. 
by plants-is the migration of incident light energy, captured at the periphery of a chromophore array, to a central chromophore, effectively concentrating the excitation. Traditionally, energy transfer within light-harvesting complexes is understood according to Förster RET theory, which will be introduced in the following sections. In recent years, researchers have also become interested in special circumstances where Förster theory fails to adequately describe energy transfer, since these cases are likely to inspire new applications and will certainly indicate new physical concepts.

\subsection{HISTORY OF RET}

\subsubsection{The First Experiments}

RET, the process in which the energy of an excited atom or molecule (usually called the donor, but known historically as the "sensitizer") is transferred nonradiatively to an acceptor molecule (activator), occurs through intermolecular dipole-dipole coupling. The origins of its discovery can be traced back to 1922, when the phenomenon of RET (sensitized fluorescence) was first experimentally observed by Cario and Franck [1-3] in the gas phase. Their spectroscopic experiment involved illuminating a mixture of mercury and thallium vapors at a wavelength absorbed solely by the mercury; the resulting fluorescence spectra proved to include frequencies that could only be emitted from thallium. Such energy transfer in vapors was at first assumed to be uniquely associated with interatomic collisions, but a discovery that transfer could occur at larger separations than the collision radius showed that this was not necessarily the case. Soon RET was also being observed in solutions [4], and over the following years in a rapidly increasing number of other physical systems.

\subsubsection{Early Developments of Theory}

The first theoretical explanation of the phenomenon was proposed by Jean Perrin [5], later a Nobel laureate. Recognizing that energy could be transferred from an excited molecule to its neighbors amongst closely spaced molecules through dipole interactions, he named this process transfert d'activation, and his paper on the subject became the earliest attempt to describe nonradiative (near-field) energy transfer. Despite its initial success, however, Perrin's model incorrectly predicted that nonradiative energy transfer should be possible between dye molecules up to an intermolecular distance of $1000 \AA$, an error deriving from an inaccurate assumption that the molecules would act as Hertzian oscillators with exactly defined resonance frequencies. Five years later [6], Perrin's son Francis developed a corresponding quantum mechanical theory of RET, based on Kallman and London's results [7]. In this work he recognized a "spreading of absorption and emission frequency" due to the interactions of the dye with the solvent, reducing the probability of energy transfer. As a result, efficient transfer was calculated to occur up to $150-250 \AA$, still approximately a factor of three more than had been experimentally observed. A detailed and highly readable survey of these early contributions of J. Perrin and F. Perrin can be found in a review by Berberan-Santos [8]. 


\subsubsection{Förster Theory}

Extending the ideas of J. Perrin and F. Perrin, Förster developed the first essentially correct theoretical treatment of RET [9-11]. Förster determined that energy transfer, through dipolar coupling between molecules, mostly depends on two important quantities: spectrum overlap and intermolecular distance. Following the observation that "the absorption and fluorescence spectra of similar molecules are far from completely overlapping," he found a means to quantify the spectral overlap integral. The static dipole-dipole interaction was known to have an inverse proportionality on the cube of the molecular separation. Since the rate of energy transfer is proportional to the square of such a coupling, it thus depends on the sixth power of the separation-that is, the now-famous $R^{-6}$ distance-dependence law. Moreover, the acceptor distance at which this rate equates to that of spontaneous emission by the donor, later termed the Förster radius $R_{0}$, was calculated to lie between 10 and $100 \AA$, agreeing with experimental observations.

Much later, the distance dependence predicted by Förster was fully verified by fluorescence studies of donor-acceptor pairs at known separations [12,13], leading to the suggested employment of RET as a "spectroscopic ruler" by Stryer and Haugland [13]; hence, a technique to measure the proximity relationships and conformational change in macromolecules was realized (see Section 3.4). With the introduction of the first lasers in the 1960s, the modern understanding of RET led to a raft of modern applications. An excellent in-depth review on the modern history of RET is given by Clegg [14].

\subsection{THE PHOTOPHYSICS OF RET}

RET is a process that is known to operate across a diverse and extensive range of physical systems, encompassing not only gases and dye solutions, but also protein complexes, doped crystals, and polymers, to name but a few. Nonetheless, at a fundamental level it is possible to identify numerous common features in the underlying photophysics.

\subsubsection{Primary Excitation Processes}

To approach the subject in detail, let us commence with the photoexcitation process that creates the conditions for RET to occur. When resonant ultraviolet or visible radiation impinges on any nonhomogeneous dielectric material, the primary result of photon absorption is the population of electronic excited states in individual atomic, molecular, or other nanoscale centers-henceforth, the latter are to be grouped together under the generic term "chromophore." Typically, such absorption is immediately followed by a rapid but partial degradation of the acquired energy, the associated losses (largely due to vibrational dissipation) ultimately to be manifest in the form of heat. This effect is reflected in the principle that the release of electronic energy by fluorescence generally occurs from the lowest vibrational level of 
(a)

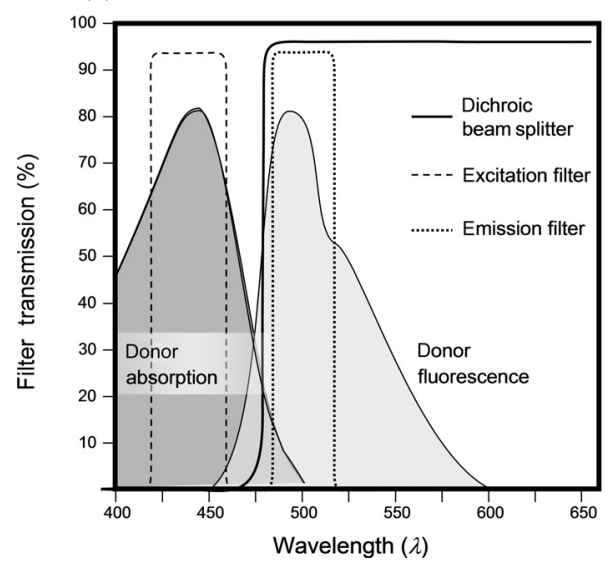

(b)

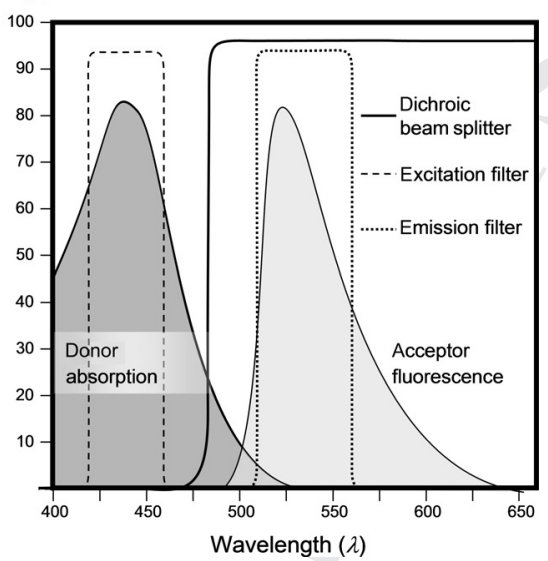

FIGURE 3.1 Typical spectral discrimination between the fluorescence from donor and acceptor species (here notionally based on a cyan fluorescent protein donor and a yellow fluorescent protein acceptor): (a) the transmission characteristics of a short-wavelength filter ensure initial excitation of only the donor; a dichroic beam splitter and another narrow emission filter ensuring that only the (Stokes-shifted) fluorescence from the donor reaches a detector; (b) in the same system a longer-wavelength emission filter ensures capture of only the acceptor fluorescence, following RET.

the excited state. However, if any nearby chromophore has a suitably disposed electronic state, of a similar or slightly lower energy, that neighbor may acquire the major part of the electronic excitation through RET - a process that takes place well before any further thermal degradation of the excited state energy occurs. The mechanism is most commonly studied through spectrometric differentiation of fluorescence emerging from the initially excited energy donor and from the energy acceptor species, as illustrated in Figure 3.1. As will be shown in the following, the propensity for energy to be transferred between any two chromophores is severely restricted by distance, and if no suitable acceptor is within reach, the donor will generally shed its energy by fluorescence or local dissipation.

\subsubsection{Coupling of Electronic Transitions}

In systems where RET occurs, the donors and acceptors are usually also fluorophores, that is, chromophores that have the capacity to decay by fluorescent emission. Moreover, in RET, the transitions of donor decay and acceptor excitation are generally electric dipole-allowed-although other possibilities do occasionally arise. Accordingly the theory of energy transfer, for donor-acceptor displacements beyond the region of significant wavefunction overlap, is traditionally conceived in terms of an electrodynamical coupling between transition dipoles.

Consider the pairwise transfer of excitation between two chromophores D and A. In the context of this elementary mechanism, D is designated the donor and $\mathrm{A}$ the 
acceptor. Specifically, let it be assumed that prior excitation of the donor generates an electronically excited species $D^{*}$. Forward progress of the energy is then accompanied by donor decay to the ground electronic state. Acquiring the energy, A undergoes a transition from its ground to its excited state. The RET process may be expressed by the following photophysical equation:

$$
\mathrm{D}^{*}+\mathrm{A} \rightarrow \mathrm{D}+\mathrm{A}^{*} .
$$

The excited acceptor, $\mathrm{A}^{*}$, subsequently decays either in a further transfer event, or by another means such as fluorescence. As the $\mathrm{D}^{*}$ and $\mathrm{A}^{*}$ excited states are real, with measurable lifetimes, the core process of energy transfer itself is fundamentally separable from the initial electronic excitation of $\mathrm{D}$ and the eventual decay of $\mathrm{A}$; the latter processes do not, therefore, enter into the theory of the pair transfer.

Since Förster theory explicitly relies on the electric dipole approximation, that is, energy transfer occurring through electric dipole couplings, the model breaks down in certain circumstances and becomes invalid. Consequently, problems of interpretation can arise when an electronic transition is electric dipole-forbidden, but allowed via magnetic or higher-order multipoles; weak interactions may develop, to facilitate the energy transfer, that are unaccounted for by the Förster theory. This scenario often occurs for interactions between highly symmetric chromophores. In the Taylor series expansion of the Coulombic interaction between transition moments, the leading nonzero term involves electric dipole moments while the higher orders relate to magnetic dipoles, and to higher electric and magnetic multipoles. All of these additional terms are more strongly dependent on distance and, therefore, multipolar interactions are significant at very small donor-acceptor separations; an alternative mechanism described by Dexter theory [15] should be considered when there is donor-acceptor wavefunction overlap (vide infra).

\subsubsection{Dissipation and Line Broadening}

To delve more deeply into the nature of the RET process, it needs to be recognized that Eq. (3.1) tells only part of the story, dealing as it does with only electronic excitations. In general, other dissipative processes are also engaged. In a solid, the linewidth of optical transitions manifests the influence of local electronic environments that, in the case of strong coupling, may lead to the production of phonon side-bands. Similar effects in solutions or disordered solids represent inhomogeneous interactions with a solvent or host, while the broad bands exhibited by chromophores in complex molecular systems signify extensively overlapped vibrational levels, including those associated with skeletal modes of the superstructure. In each case, the designation of RET as a "resonance" process has not only the connotation of overall energy conservation, but also conveys the sense that energy can transfer from any level within the continuum of the donor-excited state to a corresponding level in the excited state continuum of the acceptor. The overall transfer efficiency thus involves a factor known as the spectral overlap [16], which denotes a frequency-weighted integral product of the donor emission and acceptor absorption profiles, as illustrated in Figure 3.2. 


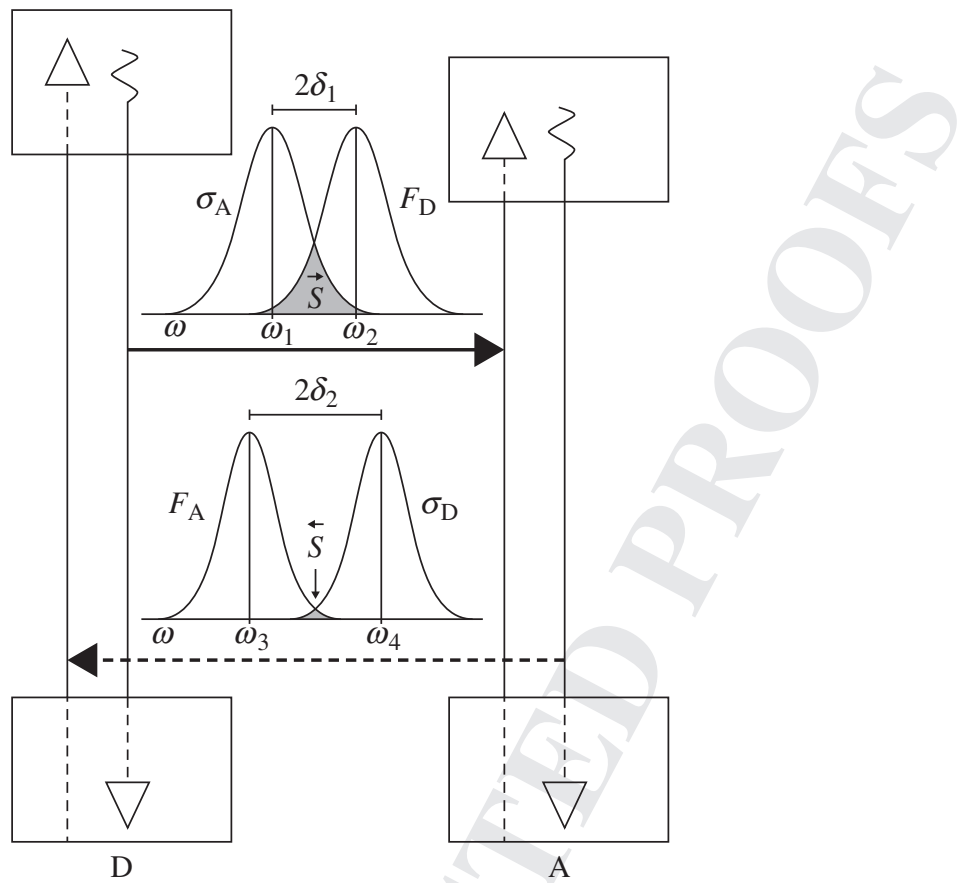

FIGURE 3.2 Energetics and spectral overlap features (top) for energy transfer from D to A (and below, potentially backward transfer from A to D). For each chromophore, $F$ denotes the fluorescence spectrum and $\sigma$ the absorption. Wavy downward lines denote vibrational dissipation.

Förster theory relies on a crucial assumption relating to the microscopic description of the dynamics, namely that the electronic coupling between donor and acceptor is very weak in comparison to line broadening. The electronic coupling acts as a small perturbation to promote energy transfer, but it is not sufficiently large to support delocalized excited states. In this weak coupling regime, vibrational equilibrium is achieved much faster than the rate at which RET occurs, and thus the electronic and vibrational states are essentially decoupled when RET takes place. When the excitation is localized on one molecule at any time and the transfer of excitation from the donor to the acceptor occurs incoherently, in an irreversible "hopping" fashion, the dynamics can be described according to conventional kinetic rate equations. Accordingly, a time-independent transfer rate can be determined from Fermi's golden rule-a quantum mechanical prescription that underpins most of molecular spectroscopy [17].

To formulate other energy transfer theories that relax this assumption is a great challenge, strongly depending on how the magnitude of the interchromophore interaction compares to the interaction of this system with the "bath"- the latter term being commonly used in this context to signify the surroundings of the donor-acceptor pair. The interchromophore interaction is characterized by direct electronic coupling, while the system-bath interaction can be characterized by a quantity called the 
"reorganization energy," which quantifies the extent to which the host or solvent and other environmental degrees of freedom accommodate the change of electron distribution on promotion of a molecule from its electronic ground to excited state. A fundamental breakdown of Förster theory occurs in the intermediate and strong coupling regimes. Here, the interchromophore coupling between donor and acceptor molecules can no longer be regarded as sufficiently small to promote incoherent energy transfer. In the strong coupling limit, when the coupling between the donor and acceptor molecules is large compared to the system-bath coupling, delocalized excitonic states are created; the excitation is coherently shared, being no longer localized on either the donor or acceptor [18]. The applicable description of the strong coupling regime is known as Redfield theory [19]. In Redfield theory, it is the bath that promotes energy transfer and relaxation through stochastic fluctuations.

In the intermediate coupling regime, widely applicable to multichromophore lightharvesting proteins, the electronic coupling is similar in magnitude to the system-bath coupling. Such cases, in which the understanding of energy transfer becomes far more difficult, are currently the subject of extensive studies [20].

\subsubsection{Förster Equation}

The Förster theory delivers an expression for the rate of pairwise energy transfer, $w_{\mathrm{F}}$, for any donor-acceptor separation, $R$, that is substantially smaller than the wavelengths of visible radiation. For systems where the common host material for the donor and acceptor has refractive index $n$, at an optical frequency corresponding to the mean transferred energy, the Förster result is expressible as follows [21-23]:

$$
w_{\mathrm{F}}=\frac{1}{\tau_{\mathrm{D}}^{0}}\left[\frac{R_{0}}{R}\right]^{6},
$$

where $\tau_{\mathrm{D}}^{0}$ is the radiative decay lifetime of the donor molecule in the absence of transfer. The Förster radius $R_{0}$ (in Ångstroms) - that is, the distance at which the rates of donor deactivation by RET and by spontaneous fluorescence become equal-is defined by

$$
R_{0}=0.2108\left[\kappa^{2} \Phi_{\mathrm{D}}^{0} n^{-4} J\right]^{\frac{1}{6}} .
$$

In this expression, $\Phi_{\mathrm{D}}^{0}$ is the fluorescence quantum yield of the donor with transfer absent, $\kappa$ is the orientation factor (vide infra), and $J$ represents an integral of the product of the donor emission spectrum with the acceptor absorption spectrum (in units of $\mathrm{M}^{-1} \mathrm{~cm}^{-1} \mathrm{~nm}^{4}$ ); the latter denotes a spectral overlap which, as stated earlier, is a key determinant of energy transfer efficiency. It is evident from Figure 3.2, that the propensity for forward transfer is usually significantly greater than that for backward transfer, due to a sizeable difference in the spectral overlaps for the two processes. Similar to the rate, the transfer efficiency $\Phi_{\mathrm{T}}$ is expressible as

$$
\Phi_{\mathrm{T}}=\frac{1}{1+\left(R / R_{0}\right)^{6}}=1-\frac{\tau_{\mathrm{fl}}}{\tau_{\mathrm{D}^{*}}}=1-\frac{I_{\mathrm{fl}}}{I_{\mathrm{D}^{*}}},
$$




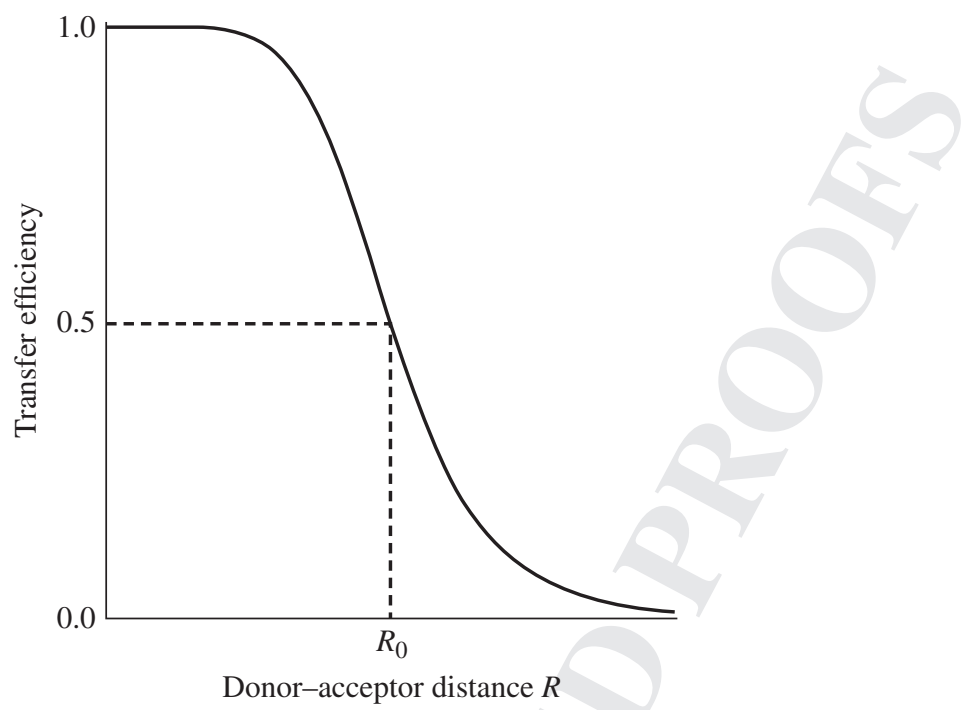

FIGURE 3.3 Distance dependence of the transfer efficiency between a pair of chromophores, calculated according to Eq. (3.4).

where $I_{\mathrm{fl}}$ and $I_{\mathrm{D}^{*}}$ are the intensities of the donor fluorescence with the acceptor present and excluded, respectively, and $\tau_{\mathrm{fl}}$ specifically denotes the fluorescence lifetime of the donor measured within its RET environment. As graphically depicted in Figure 3.3, a donor-acceptor displacement equal to $R_{0}$ corresponds to a transfer efficiency of $50 \%$.

The third equality on the right of Eq. (3.4), which holds provided decay processes follow single-exponential decay kinetics, provides a formula that is cast in terms of easily measurable quantities. This is particularly useful since it allows energy transfer efficiencies to be calculated simply on the basis of intensity measurements (e.g., using a fluorimeter), obviating the separate time-resolved measurements that would otherwise be generally necessary for evaluation of the characteristic decay lifetimes $\tau_{\mathrm{fl}}$ and $\tau_{\mathrm{D}^{*}}$. When a given electronically excited chromophore is situated within a distance $R_{0}$ of a suitable acceptor, RET will generally be the dominant decay mechanism; conversely, for distances beyond $R_{0}$, spontaneous decay (usually fluorescence) will be the primary means of donor deactivation.

\subsubsection{Orientation Dependence}

The $\kappa$ factor depends on the orientations of the donor and acceptor, both with respect to each other, and with respect to their mutual displacement unit vector $\hat{\boldsymbol{R}}$, as follows:

$$
\kappa=\left(\hat{\mu}_{\mathrm{D}} \cdot \hat{\boldsymbol{\mu}}_{\mathrm{A}}\right)-3\left(\hat{\boldsymbol{R}} \cdot \hat{\boldsymbol{\mu}}_{\mathrm{D}}\right)\left(\hat{\boldsymbol{R}} \cdot \hat{\boldsymbol{\mu}}_{\mathrm{A}}\right) .
$$

For each chromophore, $\hat{\mu}$ designates a unit vector in the direction of the appropriate transition dipole moment - a measure of charge displacement during the associated transition, which can be calculated using quantum theoretical methods [24]. The 


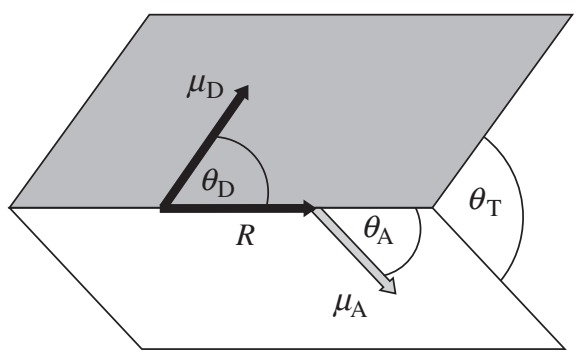

FIGURE 3.4 Relative orientations and positions of the donor and acceptor and their transition moments: angles $\theta_{\mathrm{D}}$ and $\theta_{\mathrm{A}}$ are subtended by donor and acceptor transition moments $\left(\mu_{\mathrm{D}}\right.$ and $\boldsymbol{\mu}_{\mathrm{A}}$, respectively) against the interchromophore displacement vector, $\mathbf{R}$; the symbol $\theta_{\mathrm{T}}$ is the angle between the transition moments.

possible values of $\kappa^{2}$, as featured in Eq. (3.3), lie in the range (0, 4). It is evident that in the case of fixed chromophore positions and orientations the result delivered by (3.5) is a function of three independent angles, as shown and defined in Figure 3.4:

$$
\kappa=\cos \theta_{\mathrm{T}}-3 \cos \theta_{\mathrm{D}} \cos \theta_{\mathrm{A}} .
$$

Unfavorable orientations can thus reduce the rate of energy transfer to zero; other configurations, including many of those found in photobiological systems, optimize the transfer rate. The angular disposition of chromophores is therefore a very important facet of energy transfer. It is important to note that transfer is not necessarily precluded when the transition moments lie in perpendicular directionsprovided that neither is also disposed orthogonally to $\boldsymbol{R}(=R \hat{\boldsymbol{R}})$.

In any at least partially fluid or disordered system, the relative orientation of all donor-acceptor pairs may not be identical, and it is then the distributional average of $\kappa^{2}$ that determines the overall measured response. In the isotropic case (completely uncorrelated orientations) the $\kappa^{2}$ factor averages to $2 / 3$; departures from this value provide a quantitative signature of the degree of orientational correlation. In molecules of sufficiently high symmetry it can also happen that either the donor or the acceptor transition moment is not unambiguously identifiable with a particular direction in the corresponding chromophore reference frame. Specifically, the electronic transition may then relate to a transition involving a degenerate state-as can occur with square planar complexes, for example [25]. Alternatively, the same observational features might indicate rapid but orientationally confined motions. The considerable complication which each of these effects brings into the trigonometric analysis of RET has been extensively researched and reported by van der Meer [26, 27].

\subsubsection{Polarization Features}

When linearly polarized laser light is used to excite any specific species within a complex disordered solid or liquid system, the probability for initial excitation of any particular molecule is proportional to $\cos ^{2} \theta$, where $\theta$ is the angle between the 
appropriate excitation transition moment and the electric polarization vector of the input radiation. Consequently the population of excited molecules has a markedly anisotropic distribution, a phenomenon associated with the term photoselection. If radiative decay were to ensue instantaneously, that is, from precisely the initially populated excited level, then the fluorescence would carry the full imprint of that anisotropy and itself exhibit a degree of polarization - the highest value possible. Accounting for the necessary three-dimensional rotational average [28], it is readily shown that the fluorescence intensity components polarized parallel to and perpendicular to the polarization of the excitation beam, $I_{\|}$and $I_{\perp}$, respectively, would then lie in the ratio $3: 1$. Commonly observed departures from this result thus signify the extent to which the orientation of the emission dipole differs from that of the prior, initial excitation - which may be due to intervening decay, molecular motion, or intermolecular energy transfer.

The two most widely used quantitative expressions of polarization retention are the fluorescence anisotropy, $r$, or the degree of polarization, $P$. Both convey the same information; they are defined and related as follows:

$$
r=\frac{I_{\|}-I_{\perp}}{I_{\|}+2 I_{\perp}}, \quad P=\frac{I_{\|}-I_{\perp}}{I_{||}+I_{\perp}} \quad \Rightarrow \quad r=\frac{2 P}{3-P} .
$$

The denominator of the expression for $r$ designates the net fluorescence intensity. In a specific situation where the donor and acceptor have transition dipole moments oriented in parallel, then $r=0.4$ and $P=0.5$.

A key molecular factor determining any loss in polarization is the angle $\theta$ between the directions of the absorption and emission transition dipole moments. In terms of this parameter and its influence on the measured fluorescence anisotropy, the case where internal decay intervenes between excitation and fluorescence decay within a single molecule is no different from that of a donor-acceptor pair in which the absorption and emission processes are spatially separated - provided the donor and acceptor in the latter case have a fixed mutual orientation (the orientation of the pair being random). The following result, derived by Levshin [29] and Perrin [30], can be applied in both situations:

$$
P=\frac{3 \cos ^{2} \theta-1}{3+\cos ^{2} \theta} .
$$

In the case of a donor-acceptor pair, $\theta$ is to be interpreted as the angle $\theta_{\mathrm{T}}$ shown in Figure 3.4. Equation (3.8) thus allows direct calculation of this microscopic parameter, through measurement of the macroscopic quantity $P$. Moreover when $P$ proves to exhibit a time-dependent decay, a study of the kinetics provides information on the extent of rotational motion intervening between the absorption and emission events.

Very different behavior is observed for RET systems in which the donor and acceptor are orientationally uncorrelated, that is, where they are both, independently, randomly oriented. In such cases there is a very rapid loss of polarization "memory," and it transpires that the associated degree of anisotropy is precisely $1 / 25$, that is, $r=0.04$ [31]; two or more energy transfer jumps will therefore usually, to all intents 
and purposes, destroy any polarization in any ensuing fluorescence. However, it should be noted that there is a surprising recovery in the anisotropy at distances approaching the transfer wavelength. The effect is sufficiently strong to warrant attention in dilute solution studies [32].

\subsubsection{Diffusion Effects}

So far only RET between a donor-acceptor pair has been considered. The discussion is now extended to an ensemble of donors D and acceptors A, all units of which are distributed randomly within an $m$-dimensional volume. For systems in which translational diffusion is extremely slow compared to the rate of energy transfer, the time dependence of the donor intensity decay at time $t, I_{\mathrm{D}^{*}}(t)$, as obtained by Förster [11], is given by the following expression:

$$
I_{\mathrm{D}^{*}}(t)=I_{\mathrm{D}^{*}}(0) \exp \left[-\frac{t}{\tau_{\mathrm{D}^{*}}}-2 \gamma\left(\frac{t}{\tau_{\mathrm{D}^{*}}}\right)^{\frac{m}{6}}\right] .
$$

The most commonly applied form of this expression is when the $m$ equals 3 , that is, RET in three dimensions. In Eq. (3.9), the parameter $\gamma$ is explicitly written as

$$
\gamma=\frac{2}{3} \pi^{\frac{3}{2}} C_{\mathrm{A}} R_{0}^{3}
$$

in which $C_{A}$ is the concentration of acceptors (number per unit volume) and (4/3) $\pi R_{0}^{3} C_{\mathrm{A}}$ represents the average number of acceptor chromophores in a sphere of radius $R_{0}$; the orientational factor is again set as $2 / 3$.

Cases where diffusion is comparable to the transfer rate become very complicated, and calculations by Butler and Pilling [33] have shown that large errors arise on using Förster theory for systems with diffusion coefficients in excess of $10^{-5} \mathrm{~cm}^{2} \mathrm{~s}^{-1}$. To address such systems, a successful approximation was developed by Gösele et al. [34]. This approach involves the insertion of a multiplier $G$ within the second term in the exponential of Eq. (3.9). With $m=3$, the parameter $G$ is given by

$$
G=\left(\frac{1+5.47 x+4.00 x^{2}}{1+3.34 x}\right)^{\frac{3}{4}}
$$

in which $x=D\left(R_{0}^{6} / \tau_{\mathrm{D}^{*}}\right)^{-1 / 3} t^{2 / 3}$, where $D$ is the mutual diffusion coefficient. In contrast to the Förster theory, the above method provides an excellent approximation-as was verified by the authors of Reference 33 .

Förster theory also fails at donor-acceptor displacements similar in magnitude to chromophore dimensions for another reason. This is because transition dipole moments no longer appear point-sized, and the centers of the transition moments, from which charge displacement is measured, become difficult to define. Circumstances where this is especially apparent are extended chromophores such as carotenoid, chlorophyll, and other photosynthetic substances. An expedient solution to the problem is the transition density cube (TDC) method, which involves explicit calculations 
of the interactions between relevant transition densities on the donor and acceptor [35]. The key advance of transition densities over their approximation via the Taylor expansion is that exact Coulombic coupling is determined, that is, all the terms in the expansion are retained, and the wavefunction shapes of the interacting chromophores are captured. In fact the shapes of the transition densities (which are related to the wavefunctions) are vital in understanding the molecular interactions. In its original form, the TDC method uses quantum mechanical computations to obtain the transition densities between ground and excited states. These are discretized into infinitesimal volume elements, followed by a summation over all Coulombic interactions. Higher order energy transfer processes can occur via electric quadrupole-electric quadrupole interactions or second-order electric dipole-electric dipole couplings [36].

\subsubsection{Long-Range Transfer}

It was originally assumed that a "radiative" mechanism would correctly describe energy transfer for donor-acceptor separations over and beyond $100 \AA$, and some recent literature on the subject still perpetuates this over-statement. Certain sources wrongly treat Förster "radiationless" energy transfer as exact, distinct, and separable from "radiative" energy transfer-the latter signifying successive but independent processes of fluorescence emission by a donor, and capture of the ensuing photon by an acceptor.

Although that certainly is the observed character of RET over very long distancesas for example between donor and acceptor components in a dilute solution-it is now known that both "radiative" and Förster transfer are simply the long- and short-range limits of one powerful, all-pervasive mechanism. The latter, determined from quantum electrodynamical calculations, is the outcome of the unified theory of RET [37]. This not only embraces Förster and "radiative" energy transfer, but also addresses the intermediate range in which neither of these mechanisms is fully valid. An expression for the total pairwise energy transfer rate, ranging from molecular dimensions up to interstellar distances, is written as

$$
w=w_{\mathrm{F}}+w_{\mathrm{I}}+w_{\mathrm{rad}},
$$

where $w_{\mathrm{F}}$ represents the Förster rate of Eq. (3.2), equally expressible in an alternative form as follows [38,39]:

$$
w_{\mathrm{F}}=\frac{9 \kappa^{2} c^{4}}{8 \pi \tau_{\mathrm{D}}^{0} n^{4} R^{6}} \int F_{\mathrm{D}}(\omega) \sigma_{\mathrm{A}}(\omega) \frac{d \omega}{\omega^{4}},
$$

where $F_{\mathrm{D}}(\omega)$ denotes the normalized fluorescence spectrum of the donor, $\sigma_{\mathrm{A}}(\omega)$ represents the linear absorption cross-section of the acceptor, and $\omega$ is an optical frequency in radians per unit time. Returning to Eq. (3.12), $w_{\text {rad }}$ is the rate of "radiative" energy transfer-explicitly given by

$$
w_{\text {rad }}=\frac{9 \kappa^{\prime 2}}{8 \pi \tau_{\mathrm{D}}^{0} R^{2}} \int F_{\mathrm{D}}(\omega) \sigma_{\mathrm{A}}(\omega) d \omega,
$$


and $w_{\mathrm{I}}$ is the intermediate term that is cast as

$$
w_{\mathrm{I}}=\frac{9 c^{2}}{8 \pi \tau_{\mathrm{D}}^{0} n^{2} R^{4}}\left(\kappa^{2}-2 \kappa \kappa^{\prime}\right) \int F_{\mathrm{D}}(\omega) \sigma_{\mathrm{A}}(\omega) \frac{d \omega}{\omega^{2}} .
$$

In both Eqs. (3.14) and (3.15), the symbol $\kappa^{\prime}$ denotes an orientation factor identical to (3.5) but with the factor of " 3 " omitted from the second term. In summary, the unified theory of RET contains not only the $R^{-6}$ term of Förster theory and the $R^{-2}$ term denoting the inverse-square law of "radiative" transfer, but also a previously unidentified $R^{-4}$ intermediate term.

\subsubsection{Dexter Transfer}

Before concluding this section, it is worth observing that other forms of donoracceptor coupling are also possible, although considerably less relevant to the systems of interest in the following account of applications. For example, the transfer of energy between atomic components with significantly overlapped wavefunctions is usually described in terms of Dexter theory [40]—-where the coupling involves electron exchange and is associated with an exponential decay with distance, directly reflecting the radial form of overlapping wavefunctions and electron distributions. Unlike Förster transfer, singlet-triplet energy exchange $\left({ }^{3} \mathrm{D}^{*}+{ }^{1} \mathrm{~A} \rightarrow{ }^{1} \mathrm{D}+{ }^{3} \mathrm{~A}^{*}\right)$ may also be allowed by the Dexter mechanism. This is because Dexter transfer, which is not simply expressible in terms of transition moments, is not precluded by the dipoleforbidden character of the transitions $T_{1} \rightarrow S_{0}$ and $S_{0} \rightarrow T_{1}$ within chromophores D and A, respectively. The Dexter mechanism requires only the conservation of total spin. Compared to materials in which the donor and acceptor orbitals do not spatially overlap, such systems are of less use for either device or analytical applications. The Dexter mechanism is generally operational only at very short distances $(<10 \AA)$, and other terms dominate the electronic coupling in cases of orbital overlap. The reader is referred to References 15 and 41 for relevant discussions.

\subsection{INVESTIGATIVE APPLICATIONS OF RET IN MOLECULAR BIOLOGY}

Without doubt, the field in which measurements of RET have had the greatest impact is molecular biology. The importance of RET to this subject, especially in application to biological macromolecules, was first realized following the construction of spectroscopic equipment for routine fluorescence measurements [42-45]. Toward the turn of the twenty-first century, RET underwent a period of significant redevelopment as a spectroscopic technique [46]. This resurgence arose mainly due to the advent of new experimentation methods, for example, single-pair RET [47], and further advances in instrumentation. The key advantage of RET techniques over others is that fluorescence measurements are highly sensitive, being made against a zero background; moreover, the UV/visible signals are relatively easy to detect and are specific and the required instrumentation is noninvasive. 


\subsubsection{Spectroscopic Ruler}

A major use of RET, based on its strong distance dependence, exploits its capacity to supply accurate spatial information about molecular structures. This derives from a quantitative assessment of the interchromophore separations, based on comparisons between the corresponding RET efficiencies [48-51]. Such a technique is popularly known as a "spectroscopic ruler." The elucidations of molecular structure by such means usually lack information on the relative orientations of the groups involved, and as an expedient the calculations usually ignore the kappa parameter (3.4). The apparent crudeness of this approach becomes more defensible on realizing that, even if it were to introduce a factor of two inaccuracy, the deduced group spacing would still be in error by only $12 \%$ (since $2^{1 / 6}=1.12$ ). Refinements to the theory to accommodate the effect of fluctuations in position or orientation of the participant groups introduce considerable complexity, although progress is being made in several areas [52-56].

\subsubsection{Conformational Change}

Through identification of motions in macromolecules, that is, the variation in proximity of one chromophore with respect to another, a number of valuable RET applications arise, including the detection of conformational changes and folding in proteins [49,57-59], and the inspection of intracellular protein-protein [24, 60-63] and protein-DNA $[64,65]$ interactions (see, e.g., Figure 3.5). These and other such processes can be registered by selectively exciting one chromophore using laser light and monitoring either the decrease in fluorescence from that chromophore, or the rise in the generally longer-wavelength fluorescence from the other chromophore as it adopts the role of acceptor. The judicious use of optical dichroic filters can make this RET technique perfectly straightforward-see Figure 3.1. In cases where the two material components of interest lack suitably overlapped absorption and fluorescence

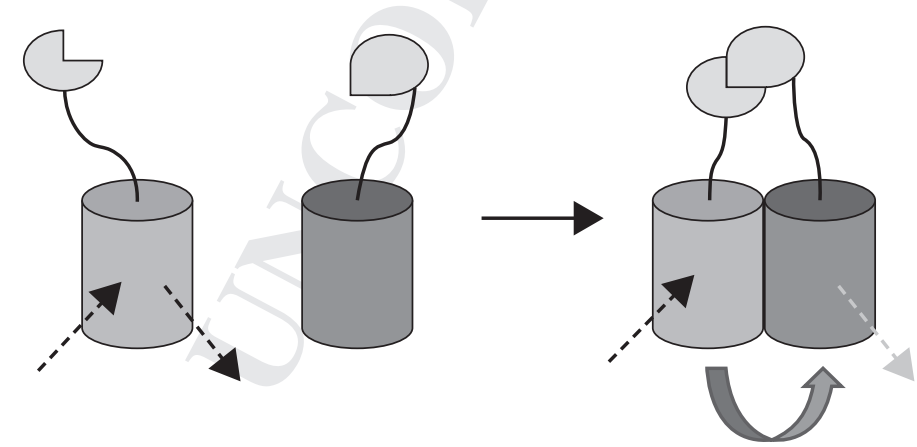

FIGURE 3.5 RET method for the detection of protein-protein interactions. Biochemical interactions between the proteins result in the attached chromophores becoming closer in proximity-thus allowing energy transfer to occur between them. As a consequence, the emission frequency will differ to those cases where energy transfer is absent. 
features in an optically accessible wavelength range, molecular tagging with sitespecific "extrinsic" (i.e., artificially attached) chromophores can solve the problem. Located at a molecular site of interest, and being selected on the basis of a significant spectral overlap with the counterpart component, such tags can act either in the capacity of donor or acceptor. Lanthanide ions, with their characteristically prominent and line-like absorption features, prove particularly valuable in this connection [66]. Also useful in this respect are the semiconductor nanocrystals known as quantum dots. These crystalline nanoparticles offer several unique traits, including size- and composition-tunable emission from visible to infrared wavelengths, the possibility of a single light source simultaneously exciting different-sized dots, large absorption coefficients across a wide spectral range, and very high level of photostability $[67,68]$.

\subsubsection{Intensity-Based Imaging}

In the last two decades, there has been burgeoning interest in microscopy based on RET [69-75], typical instrumentation for which is illustrated in Figure 3.6. There are three specific types of RET method routinely used in the production of biological images. The principles of sensitized-emission RET have already been described (Fig. 3.1). For microscopy purposes this method is fairly inaccurate; no RET donoracceptor pair is ideal, that is, there will almost always be some overlap between the donor and acceptor absorption bands, and also the donor and acceptor emission

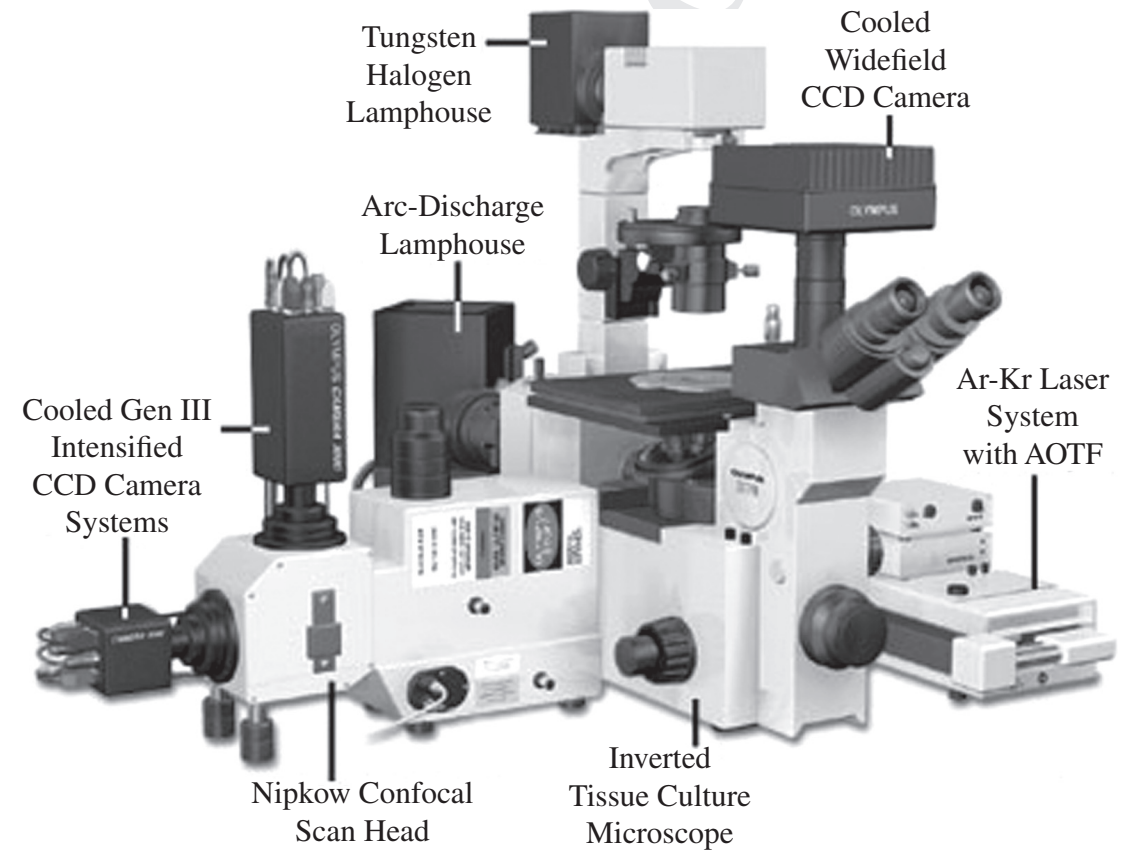

FIGURE 3.6 Typical commercial RET microscope (Olympus Corporation). 
spectra. Therefore, filters that completely separate these kinds of spectrum are difficult to design. Various calculational algorithms [76-78] have been proposed to compensate for this problem, although the methods are complex and no single procedure has received universal acceptance.

A widely used alternative, experimental approach $[79,80]$ involves deliberately photobleaching the acceptor, the result of which is complete exclusion of RET. In this method, the donor emission is analyzed before and after the acceptor is bleached by the input of an intense laser beam (at a suitable wavelength). The difference between the donor intensities, with and without the laser input, enables a determination of the transfer efficiency by employing Eq. (3.4). Here, account is taken of spectral bleed-through between the two absorption bands, and equally between the two emission bands. Signal contamination is still not entirely eliminated, due to a small amount of back-transfer through donor excitation by acceptor emission. Often the main disadvantage in prolonged illumination of the acceptor is the possibility of damage to the sample. Therefore, in practice, photobleaching is seldom appropriate for in vivo studies.

\subsubsection{Lifetime-Based Imaging}

Fluorescence need not be characterized from excitation and emission spectra alone; highly significant information can also be secured from lifetime measurements. Thus, when suitable time-resolved instrumentation is available, the determination of decay kinetics (usually on the nanosecond timescale) enables analysis through RET-based fluorescence lifetime imaging microscopy (FRET-FLIM) [81-84]. In this method, spectral bleed-through is no longer an issue since measurements are made only for the determination of donor lifetimes; back-transfer is usually extremely low and within the noise level. The presence of the acceptor within the local environment of the donor influences the fluorescence lifetime of the donor. By measuring the donor lifetime in the presence and absence of the acceptor one can accurately calculate the transfer efficiency by the use of Eq. (3.4). Drawbacks to FRET-FLIM are the technical challenges the technique presents, and the expense of the equipment. Nonetheless, in optical systems that are equipped to provide both intensity and lifetime measurements, a comparison of the two types of image affords a particularly rich source of information, as illustrated by the cancerous cell images of Figure 3.7.

\subsubsection{Other Applications}

Beyond the realm of molecular biology, RET has value in a number of more specifically chemical applications. In connection with quantum dots of variable size, the viable operation of a biomimetic scheme known as a "nanofountain" (Fig. 3.8) has been proven experimentally by Ohtsu's group [85]. Other prominent examples are found in the fields of synthetic macromolecules and chemical sensors. In polymer science, building on the pioneering principles of Morawetz [86], RET is now used to determine morphological information on polymer interfaces. Such studies have, for instance, enabled the quantitative characterization of interfacial thickness in polymers 


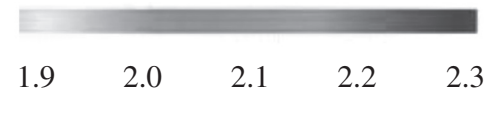

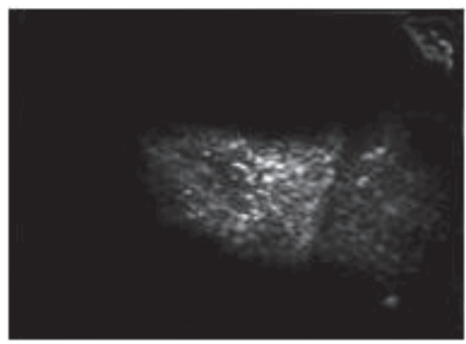

(a)

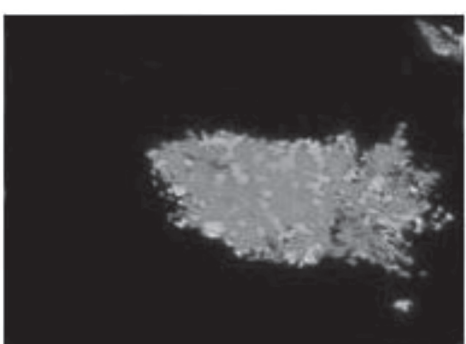

(b)

Au: Please FIGURE 3.7 MDA-MB-231 cancerous cell images recorded with argon laser two-photon check whether excitation, RET microscope based on (a) intensity and (b) fluorescence lifetime (ns). In the permission has latter, areas of locally reduced lifetime signify clustered intracellular vesicles. Adapted from
been obtained for Figure 3.7. Reference 85.

of various structures [87]. Moreover, RET has been utilized in the study of polymer conformational dynamics. One especially interesting application is the effective differentiation between various collapsed and/or ordered homopolymer chain conformations through the associated distribution of transfer efficiencies [88, 89].

The fabrication of RET-based, analyte-specific sensors has enabled detection of a variety of species, including dimers of functionalized calixarenes in organic solutions [90], copper(II) in aqueous solution [91], hydrogen peroxide [92], phosgene [93], and many others. These chemical sensors usually work on the principle of a donoracceptor system designed such that the presence of the analyte causes the acceptor chromophore to move within closer proximity to a donor, enabling an RET process that is not observed in the absence of the analyte. Therefore, on irradiation of the system with the relevant chemical present, a strong emission from the acceptor signals the presence of the analyte.

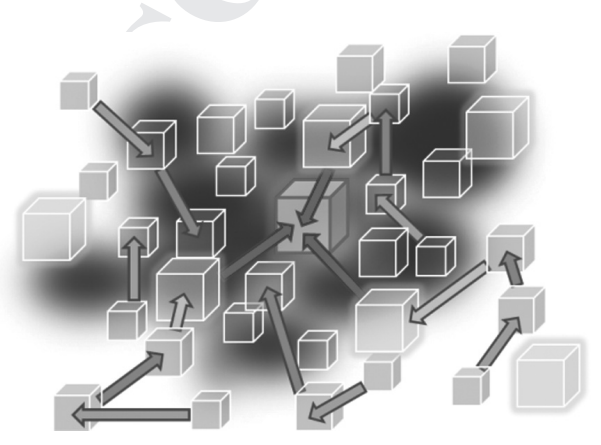

FIGURE 3.8 Scheme for an optical nanofountain composed of $2-10 \mathrm{~nm} \mathrm{CuCl}$ quantum dots, distributed in an $\mathrm{NaCl}$ matrix. (For a color version of this figure, see the color plate section.) 


\subsection{THE ROLE OF RET IN LIGHT-HARVESTING COMPLEXES}

\subsubsection{Introduction}

Light-harvesting proteins play the functional role of capturing solar radiation and transferring the resulting excitation to the reaction centers where they are used to carry out photosynthetic chemical reactions [94]. In high-order plants and many algae, the major light-harvesting protein is Light-Harvesting Complex II (LHC-II) which contains the green pigment chlorophyll. In addition to chlorophyll-containing complexes, some plants, algae, and bacteria utilize other light-harvesting antennae including phycobilisomes and phycobiliproteins to supplement the absorption of sunlight. These peripheral light-harvesting proteins transfer photoexcitation to chlorophyll- $a$ molecules located in the membrane, which subsequently pass the excitation on to photosystem I (PSI) and photosystem II (PSII). At the center of the PSII complex resides the reaction center, the site of water oxidation catalyzed by a $\mathrm{Mn}_{4} \mathrm{O}_{x} \mathrm{Ca}$ complex. The oxidation of water by PSII, along with processes carried out by other protein complexes including PSI, mobile electron carriers, and the cytochrome bf complex, complete the photosynthetic cycle. These other processes include the release of molecular oxygen, the reduction of nicotinamide-adenine dinucleotide phosphate (NADP+) and adenosine diphosphate (ADP), as well as the generation of a proton gradient across the membrane.

Photosynthesis starts with the absorption of light by a chromophore, such as chlorophyll, in a light-harvesting protein, resulting in an electronic transition from the ground state to an excited state. The excited state is short-lived, relaxing to the ground state after a mere nanosecond, so that the first events of photosynthesis involving the transport of this electronic excitation to reaction centers must be ultrafast [95]. The requirement of ultrafast energy migration beyond a nearest neighbor means that these systems are often highly optimized multi-chromophore complexes. The optimization lies in the structure of the light-harvesting complexes that bind the chromophores. Figure 3.9 shows absorption spectra recorded for several such complexes, illustrating their broad spectral cross-sections, often specialized in a particular wavelength range. Figure 3.10 shows structural models elucidated from X-ray crystallography for two light-harvesting complexes. The extraordinary density of chromophores is notable, particularly in LHC-II, the major light-harvesting complex of higher plants and green algae.

Photosynthetic organisms achieve numerous advantages by employing different complexes to harvest sunlight and to drive chemical reactions [95]: (a) Lightharvesting proteins are able to increase the spatial and spectral cross-section for the absorption of sunlight without being costly to the organism, in many photosynthetic species the reaction center may be serviced by tens of light-harvesting proteins; (b) the wide variety of these complexes allows individual species the ability to survive in varying light conditions; (c) light-harvesting antennae have evolved photoprotection responses, such as down-regulation, to avoid damage in the case of excessive exposure to sunlight. 


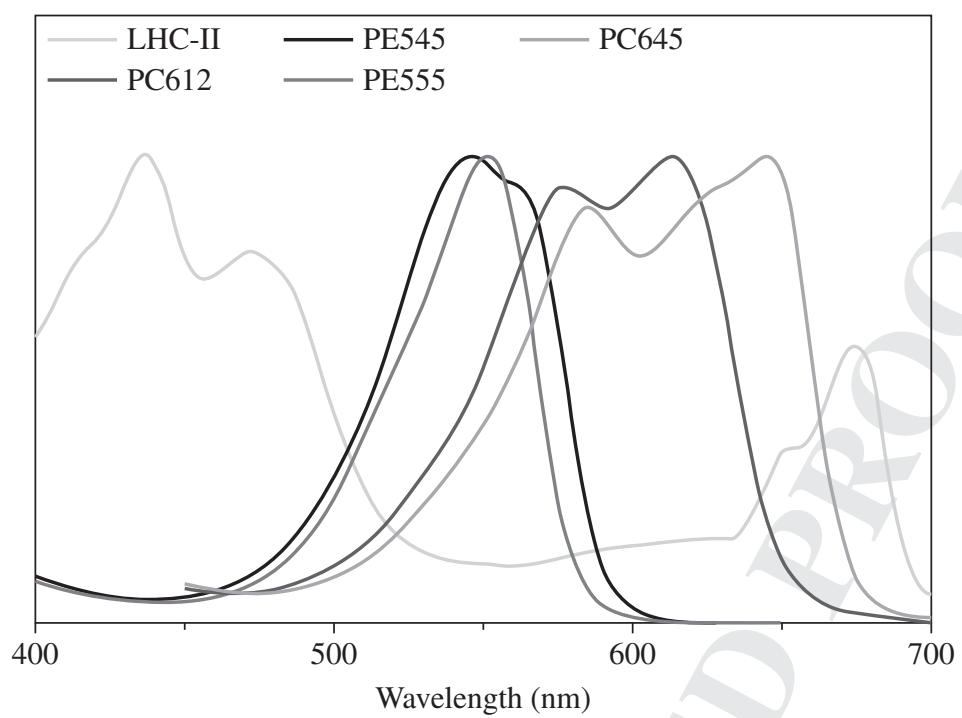

FIGURE 3.9 Normalized absorption spectrum of LHC-II (green); the main light-harvesting protein in high order plants along with the normalized absorption spectra of four cryptophyte light-harvesting proteins: PE545 (blue), PE555 (pink), PC612 (purple), and PC645 (red). The different region in which these complexes absorb is clearly evident. (For a color version of this figure, see the color plate section.)

\subsubsection{Photosynthetic Excitons}

Within a light-harvesting complex, strong electronic interactions between chromophores can result in new delocalized excited states. Although not always present, these delocalized excited states known as excitons can extend over multiple chromophores and can have a profound impact on the electronic structure and energy

(a)

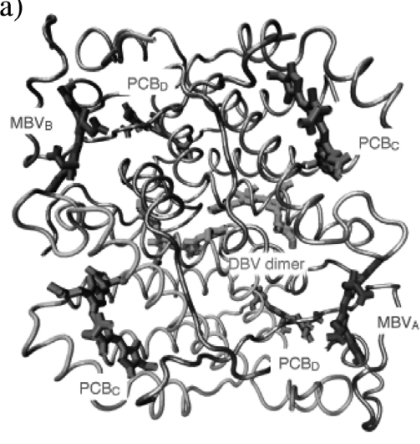

(b)

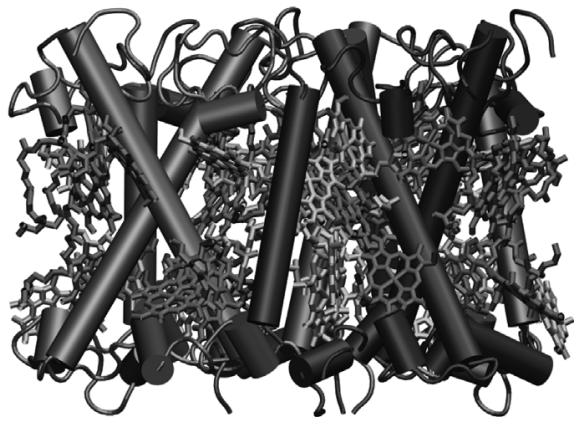

FIGURE 3.10 Structural models of two photosynthetic light harvesting complexes: (a) a cryptophyte PC645 antenna complex, and, (b) the major chlorophyll- $a / b$ complex from higher plants and green algae, LHCII. (For a color version of this figure, see the color plate section.) 


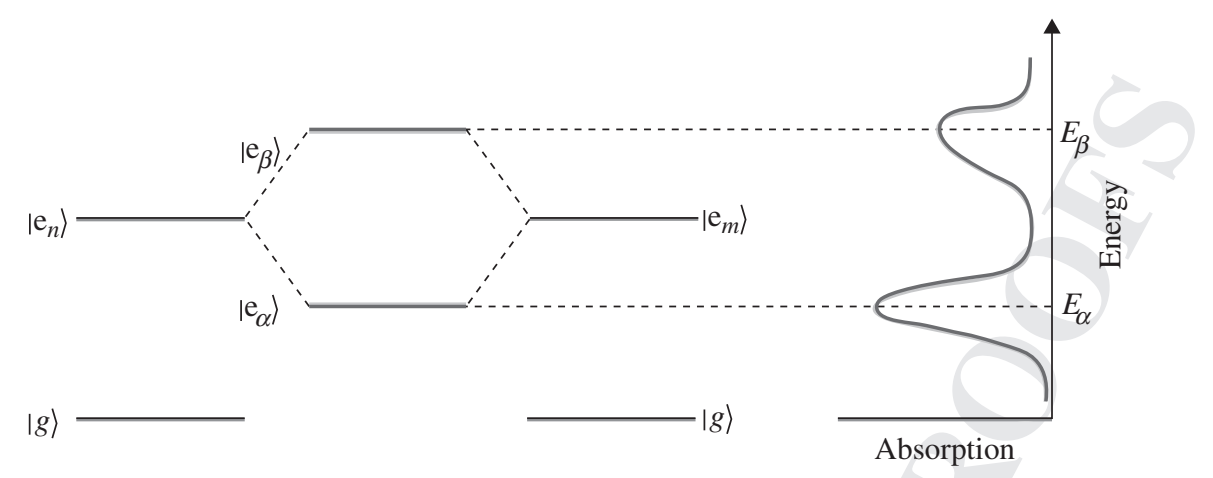

FIGURE 3.11 Illustration of the electronic energy spacing of a homodimer, which consists of two identical chromophores, each modeled as a two-level system. Molecular excited states $e_{n}$ and $e_{m}$ are coupled by an electronic potential, $V_{n m}$, resulting in delocalized excited states $e_{\alpha}$ and $e_{\beta}$. Absorption frequencies of the dimer occur at the corresponding energies $E_{\alpha}$ and $E_{\beta}$.

transfer dynamics within a light-harvesting protein. The Frenkel or molecular exciton model describes delocalized excited states in biological systems and molecular aggregates arising from the superposition of localized molecular excited states [18]. In other words, several molecules can cooperatively (in phase) absorb light and share the excitation quantum mechanically.

Delocalization of the excited state results in an increase in the spatial extent of the excited state, changing the nature of the chromophore (Fig. 3.11). The LH2 complex, isolated from purple bacteria, one of the most widely studied light-harvesting complexes, provides a clear example of how excitons can substantially change the electronic structure of a protein as well as the energy transfer dynamics [96-98]. The LH2 complex only contains one type of chromophore, bacteriochlorophyll- $a$ molecules; 27 of these molecules are arranged into two rings. In one ring, there are 18 closely packed B850 bacteriochlorophyll- $a$ molecules; due to their close proximity to each other along with their preferential orientations the coupling between these chromophores is strong, with nearest neighbor coupling being approximately $300 \mathrm{~cm}^{-1}$. In the B800 ring, there are nine loosely packed bacteriochlorophyll$a$ molecules where adjacent molecules have a $30 \mathrm{~cm}^{-1}$ electronic coupling. Photon absorption in the B800 ring occurs at $800 \mathrm{~nm}$, corresponding to electronically isolated chlorophyll- $a$ molecules, while the B850 ring is red-shifted to $850 \mathrm{~nm}$ (Fig. 3.12). This shift is in part a response to interactions between the chromophores and the protein; however, a significant contribution is due to the strong excitonic coupling of molecules. In addition to changing the electronic landscape of the system, excitons in LH2 also change the energy transfer dynamics by setting up a gradient which allows excitation to flow "downhill" from the high energy B800 ring to the lower energy B850 ring.

Förster theory for molecules cannot capture the consequences of delocalized excitation, and therefore, the unexpectedly rapid timescale for energy transfer in several light harvesting complexes were a mystery for many years. Eventually it was realized 

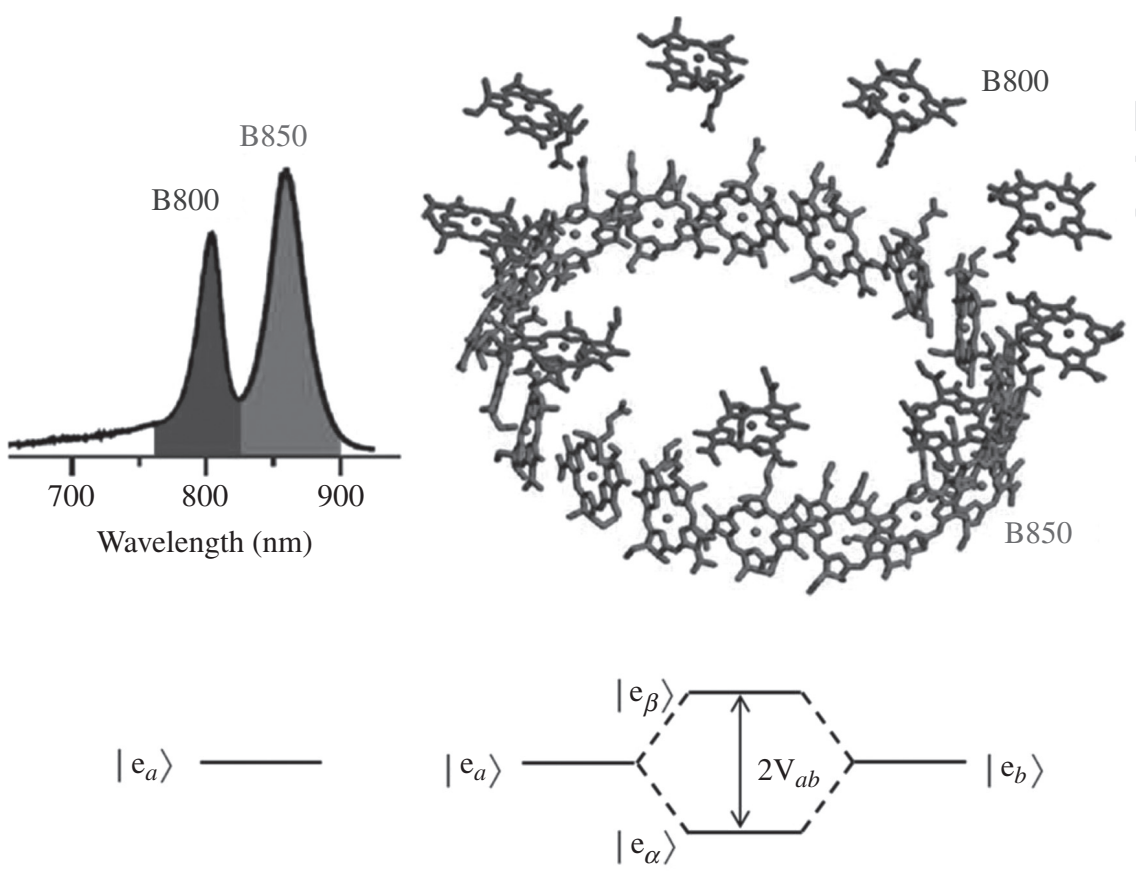

$|g\rangle$

chromophore a

$|g\rangle$
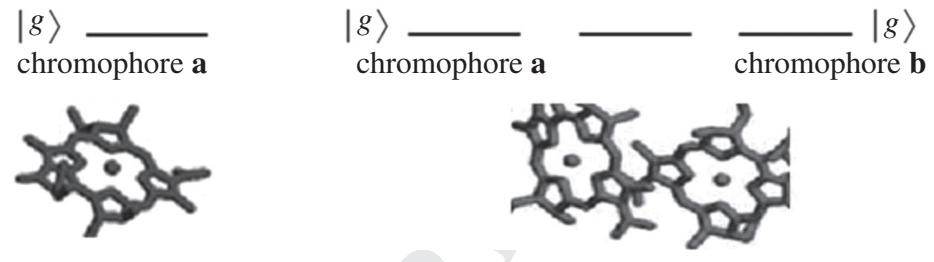

FIGURE 3.12 (a) Absorption spectrum of LH2 extracted from a strain of the purple bacterium Rhodopseudomonas acidophila. The B800 ring absorbs at $800 \mathrm{~nm}$ and is due to the nine weakly coupled $\mathrm{BChl}$ chromophores, while the $\mathrm{B} 850$ ring absorbs at $850 \mathrm{~nm}$ and is due to the 18 tightly packed BChl molecules. (b) X-ray crystallography structure of LH2 from Rps. acidophila (strain 10050) illustrating 27 bacteriochlorophyll- $a$ chromophores. Blue-colored chromophores indicate the B800 ring, while red-colored chromophores indicate the B850 ring. Energy-level diagrams for chromophores in the (c) B800 ring and (d) B850 ring. Adapted from References 10 and 41. (For a color version of this figure, see the color plate section.)

that donors and acceptors needed to be redefined in order to formulate an appropriate theory, called Generalized Förster Theory (GFT) $[99,100]$ that could quantitatively predict energy transfer in excitonic systems. The idea is to partition the system into both strongly coupled chromophores and weakly coupled chromophores, then choose effective donor and acceptor electronic states. GFT is applicable when multiple chromophores, which are strongly coupled, act together as a donor and are weakly coupled to another group of strongly coupled acceptors. 


\section{ACKNOWLEDGMENTS}

Research at the University of East Anglia is supported by the Leverhulme Trust and EPSRC. The work at the University of Toronto is supported by the Natural Sciences and Engineering Research Council of Canada and the US Air Force Office of Scientific Research (FA9550-10-1-0260).

\section{REFERENCES}

[1] J. Franck, "Einige aus der Theorie von Klein und Rosseland zu ziehende Folgerungen über Fluorscence, photochemische Prozesse und die Electronenemission glühender Körper,” Z. Phys. 9, 259-266 (1922).

[2] G. Cario, "Über Entstehung wahrer Lichtabsorption un scheinbare Koppelung von Quantensprüngen,” Z. Phys. 10, 185-199 (1922).

[3] G. Cario and J. Franck, "Über Zerlegugen von Wasserstoffmolekülen durch angeregte Quecksilberatome," Z. Phys. 11, 161-166 (1922).

[4] E. Gaviola and P. Pringsheim, "Über den einfluß der konzentration auf die polarisation der fluoreszenz von farbstofflösungen," Z. Phys. 24, 24-36 (1924).

[5] J. Perrin, "Fluorescence et induction moléculaire par résonance," C. R. Acad. Sci. 184, 1097-1100 (1927).

[6] F. Perrin, "Théorie quantique des transferts d'activation entre molecules de même espéce. Cas des solutions fluorescents," Ann. Phys. 17, 283-314 (1932).

[7] H. Kallman and F. London, "Über quantenmechanische Energieübertragung zwischen atomaren Systemen” Z. Phys. Chem. B 2, 207-243 (1928).

[8] M. N. Berberan-Santos, "Pioneering contributions of Jean and Francis Perrin to molecular luminescence," in New Trends in Fluorescence Spectroscopy. Applications to Chemical and Life Sciences, edited by B. Valeur and J.-C. Brochon (Springer, Berlin, 2001), Chap. 2.

[9] T. Förster, "Energiewanderung und fluoreszenz," Naturwissenschaften 33, 166-175 (1946).

[10] T. Förster, "Zwischenmolekulare Energiewanderung und Fluoreszenz," Ann. Phys. 2, 55-75 (1948).

[11] T. Förster, "10th Spiers Memorial Lecture. Transfer mechanisms of electronic excitation,” Discuss. Faraday Soc. 27, 7-17 (1959).

[12] S. A. Latt, H. T. Cheung, and E. R. Blout, "Energy transfer: a system with relatively fixed donor-acceptor separation," J. Am. Chem. Soc. 87, 995-1003 (1965).

[13] L. Stryer and R. P. Haugland, "Energy transfer: a spectroscopic ruler," Proc. Natl. Acad. Sci. 58, 719-726 (1967).

[14] R. M. Clegg, "The history of FRET: from conception through the labors of birth," in Reviews in Fluorescence, edited by C. D. Geddes and J. R. Lakowicz (Springer, New York, 2006), Vol. 3, Chap. 1.

[15] G. D. Scholes, "Long-range resonance energy transfer in 0molecular systems," Аnnu. Rev. Phys. Chem. 54, 57-87 (2003).

[16] D. L. Andrews and J. Rodríguez, "Resonance energy transfer: spectral overlap, efficiency and direction," J. Chem. Phys. 127, 084509 (2007). 
[17] L. Mandel and E. Wolf, Optical Coherence and Quantum Optics (University Press, Cambridge, 1995), p. 871.

[18] G. D. Scholes and G. Rumbles, "Excitons in nanoscale systems," Nat. Mater. 5, 683-696 (2006).

[19] V. May and O. Kühn, Charge and Energy Transfer Dynamics in Molecular Systems (Wiley-VCH, Weinheim, 2004).

[20] A. Ishizaki, T. R. Calhoun, G. S. Schlau-Cohen, and G. R. Fleming, "Quantum coherence and its interplay with protein environments in photosynthetic electronic energy transfer," Phys. Chem. Chem. Phys. 12, 7319-7337 (2010).

[21] J. R. Lakowicz, Principles of Fluorescence Spectroscopy, 2nd ed. (Kluwer Academic, New York, 1999), Chap. 10.

[22] B. Valeur, Molecular Fluorescence: Principles and Applications (Wiley-VCH, Weinheim, 2002), Chap. 9.

[23] S. E. Braslavsky, E. Fron, H. B. Rodríguez, E. San Román, G. D. Scholes, G. Schweitzer, B. Valeur, and J. Wirz, "Pitfalls and limitations in the practical use of Förster's theory of resonance energy transfer," Photochem. Photobiol. Sci. 7, 1444-1448 (2008).

[24] T. Ansbacher, H. K. Srivastava, T. Stein, R. Baer, M. Merkx, and A. Shurki, "Calculation of transition dipole moment in fluorescent proteins - towards efficient energy transfer," Phys. Chem. Chem. Phys. 14, 4109-4117 (2012).

[25] C. Galli, K. Wynne, S. M. Lecours, M. J. Therien, and R. M. Hochstrasser, "Direct measurement of electronic dephasing using anisotropy," Chem. Phys. Lett. 206, 493499 (1993).

[26] B. W. van der Meer, G. Coker, and S.-Y. Chen, Resonance Energy Transfer Theory and Data (VCH, New York, 1994).

[27] B. W. van der Meer, Resonance Energy Transfer, edited by D. L. Andrews and A. A. Demidov (Wiley, New York 1999), pp. 151-172.

[28] D. L. Andrews and T. Thirunamachandran, "On three-dimensional rotational averages," J. Chem. Phys. 67, 5026-5033 (1977).

[29] W. L. Levshin, "Polarisierte Fluoreszenz und Phosphoreszenz der Farbstofflosungen. IV,” Z. Phys. 32, 307-326 (1925).

[30] F. Perrin, "La fluorescence des solutions," Ann. Phys. 12, 169-275 (1929).

[31] V. M. Agranovich and M. D. Galanin, Electronic Excitation Energy Transfer in Condensed Matter (Elsevier/North-Holland, Amsterdam, The Netherlands, 1982).

[32] D. L. Andrews and G. Juzeliunas, "The range dependence of fluorescence anisotropy in molecular energy transfer," J. Chem. Phys. 95, 5513-5518 (1991).

[33] P. R. Butler and M. J. Pilling, "The breakdown of Förster kinetics in low viscosity liquids. An approximate analytical form for the time-dependent rate constant," Chem. Phys. 41, 239-243 (1979).

[34] U. Gösele, M. Hauser, U. K. A. Klein, and R. Frey, "Diffusion and long-range energy transfer," Chem. Phys. Lett. 34, 519-522 (1975).

[35] B. P. Krueger, G. D. Scholes, and G. R. Fleming, "Calculation of couplings and energy transfer pathways between the pigments of $\mathrm{LH} 2$ by the ab initio transition density cube method," J. Phys. Chem. B 102, 5378-5386 (1998).

[36] D. L. Andrews and J. M. Leeder, "Resonance energy transfer: when a dipole fails," J. Chem. Phys. 130, 184504 (2009). 
[37] D. L. Andrews, "A unified theory of radiative and radiationless molecular-energy transfer," Chem. Phys. 135, 195-201 (1989).

[38] A. A. Demidov and D. L. Andrews, in Encyclopedia of Chemical Physics and Physical Chemistry, edited by J. H. Moore and N. D. Spencer (Institute of Physics, Bristol, 2001), Vol. 3, pp. 2701-2715.

[39] D. L. Andrews, "Mechanistic principles and applications of resonance energy transfer," Canad. J. Chem. 86, 855-870 (2008).

[40] D. L. Dexter, "A theory of sensitized luminescence in solids," J. Chem. Phys. 21, 836-850 (1953).

[41] D. L. Andrews, C. Curutchet, and G. D. Scholes, "Resonance energy transfer: beyond the limits," Laser Photon. Rev. 5, 114-123 (2011).

[42] I. Z. Steinberg, "Long-range nonradiative transfer of electronic excitation energy in proteins and polypeptides," Annu. Rev. Biochem. 40, 83-114 (1971).

[43] L. Stryer, "Fluorescence energy transfer as a spectroscopic ruler," Ann. Rev. Biochem. 47, 819-846 (1978).

[44] C. G. dos Remedios, M. Miki, and J. A. Barden, "Fluorescence resonance energy transfer measurements of distances in actin and myosin. A critical evaluation," J. Muscle Res. Cell Motil. 8, 97-117 (1987).

[45] P. Wu and L. Brand, "Resonance energy transfer: methods and applications," Anal. Biochem. 218, 1-13 (1994).

[46] P. R. Selvin, "The renaissance of fluorescence resonance energy transfer," Nat. Struct. Biol. 7, 730-734 (2000).

[47] T. Ha, T. Enderle, D. F. Ogletree, D. S. Chemla, P. R. Selvin, and S. Weiss, "Probing the interaction between two single molecules: fluorescence resonance energy transfer between a single donor and a single acceptor," Proc. Natl. Acad. Sci. USA 93, 6264-6268 (1996).

[48] S. Hohng, C. Joo, and T. Ha, "Single-molecule three-color FRET," Biophys. J. 87, 1328-1337 (2004).

[49] B. Schuler, "Single-molecule fluorescence spectroscopy of protein folding," Chem. Phys. Chem. 6, 1206-1220 (2005).

[50] S. V. Koushik, H, Chen, C. Thaler, H. L. Puhl III, and S. S. Vogel, "Cerulean, venus and venus $_{\text {Y67C }}$ FRET reference standards," Biophys. J. 91, L99-L101 (2006).

[51] H. Sahoo, "Förster resonance energy transfer-a spectroscopic nanoruler: principle and applications," J. Photochem. Photobiol. C 12, 20-30 (2011).

[52] C. G. dos Remedios and P. D. J. Moens, "Fluorescence resonance energy transfer spectroscopy is a reliable 'ruler' for measuring structural changes in proteins-dispelling the problem of the unknown orientation factor," J. Struct. Biol. 115, 175-185 (1995).

[53] F. Tanaka, "Theory of time-resolved fluorescence under the interaction of energy transfer in a bichromophoric system: effect of internal rotations of energy donor and acceptor," J. Chem. Phys. 109, 1084-1092 (1998).

[54] Z. G. Yu, "Fluorescent resonant energy transfer: correlated fluctuations of donor and acceptor," J. Chem. Phys. 127, 221101 (2007).

[55] M. Isaksson, N. Norlin, P.-O. Westlund, and L. B.-Å. Johansson, "On the quantitative molecular analysis of electronic energy transfer within donor-acceptor pairs," Phys. Chem. Chem. Phys. 9, 1941-1951 (2007). 
[56] S. Jang, "Generalization of the Förster resonance energy transfer theory for quantum mechanical modulation of the donor-acceptor coupling," J. Chem. Phys. 127, 174710 (2007).

[57] D. S. Talaga, W. L. Lau, H. Roder, J. Tang, Y. W. Jia, W. F. DeGrado, and R. M. Hochstrasser, "Dynamics and folding of single two-stranded coiled-coil peptides studied by fluorescent energy transfer confocal microscopy," Proc. Natl. Acad. Sci. USA 97, 13021-13026 (2000).

[58] T. Heyduk, "Measuring protein conformational changes by FRET/LRET," Curr. Opin. Biotechnol. 13, 292-296 (2002).

[59] B. Schuler and W. A. Eaton, "Protein folding studied by single-molecule FRET," Curr. Opin. Struct. Biol. 18, 16-26 (2008).

[60] T. Zal and N. R. J. Gascoigne, "Using live FRET imaging to reveal early protein-protein interactions during T cell activation," Curr. Opin. Immunol. 16, 418-427 (2004).

[61] M. Parsons, B. Vojnovic, and S. Ameer-Beg, "Imaging protein-protein interactions in cell motility using fluorescence resonance energy transfer (FRET)," Biochem. Soc. Trans. 32, 431-433 (2004).

[62] W. B. Frommer, M. W. Davidson, and R. E. Campbell, "Genetically encoded biosensors based on engineered fluorescent proteins," Chem. Soc. Rev. 38, 2833-2841 (2009).

[63] A. W. Nguyen, X. You, A. M. Jabaiah, and P. S. Daugherty, "Fluorescent protein FRET applications," in Reviews in Fluorescence, edited by C. D. Geddes (Springer, New York, 2010), Chap. 13.

[64] A. Hillisch, M. Lorenz, and S. Diekmann, "Recent advances in FRET: distance determination in protein-DNA complexes," Curr. Opin. Struct. Biol. 11, 201-207 (2001).

[65] F. G. E. Cremazy, E. M. M. Manders, P. I. H. Bastiaens, G. Kramer, G. L. Hager, E. B. van Munster, P. J. Verschure, T. W. J. Gadella, and R. van Driel, "Imaging in situ protein-DNA interactions in the cell nucleus using FRET-FLIM," Exp. Cell Res. 309, 390-396 (2005).

[66] P. R. Selvin, "Principles and biophysical applications of lanthanide-based probes," Annu. Rev. Biophys. Biomol. Struct. 31, 275-302 (2002).

[67] W. C. W. Chan, D. J. Maxwell, X. Gao, R. E. Bailey, M. Han, and S. Nie, "Luminescent quantum dots for multiplexed biological detection and imaging," Curr. Opin. Biotechnol. 13, 40-46 (2002).

[68] A. R. Clapp, I. L. Medintz, and H. Mattoussi, "Förster resonance energy transfer investigations using quantum-dot fluorophores," Chem. Phys. Chem. 7, 47-57 (2006).

[69] R. M. Clegg, "Fluorescence resonance energy transfer," in Fluorescence Imaging Spectroscopy and Microscopy, edited by X. F. Wang and B. Herman (John Wiley \& Sons, New York, 1996), Chap. 7.

[70] A. Periasamy, Methods in Cellular Imaging (Oxford University Press, New York, 2001).

[71] F. S. Wouters, P. J. Verveer, and P. I. H. Bastiaens, "Imaging biochemistry inside cells," Trends Cell Biol. 11, 203-211 (2001).

[72] A. Hoppe, K. Christensen, and J. A. Swanson, "Fluorescence resonance energy transferbased stoichiometry in living cells," Biophys. J. 83, 3652-3664 (2002).

[73] E. A. Jares-Erijman and T. M. Jovin, "FRET imaging," Nat. Biotechnol. 21, 1387-1395 (2003). 
[74] A. Periasamy and R. N. Day, Molecular Imaging: FRET Microscopy and Spectroscopy (Oxford University Press, New York, 2005).

[75] R. N. Day and M. W. Davidson, "Fluorescent proteins for FRET microscopy: monitoring protein interactions in living cells," Bioessays 34, 341-350 (2012).

[76] G. W. Gordon, G. Berry, X. H. Liang, B. Levine, and B. Herman, "Quantitative fluorescence resonance energy transfer measurements using fluorescence microscopy," Biophys. J. 74, 2702-2713 (1998).

[77] Z. Xia and Y. Liu "Reliable and global measurement of fluorescence resonance energy transfer using fluorescence microscopes," Biophys. J. 81, 2395-2402 (2001).

[78] J. van Rheenen, M. Langeslag, and K. Jalink, "Correcting confocal acquisition to optimize imaging of fluorescence resonance energy transfer by sensitized emission," Biophys. J. 86, 2517-2529 (2004).

[79] T. S. Karpova, C. T. Baumann, L. He, X. Wu, A. Grammer, P. Lipsky, G. L. Hager, and J. G. McNally, "Fluorescence resonance energy transfer from cyan to yellow fluorescent protein detected by acceptor photobleaching using confocal microscopy and a single laser," J. Microsc. 209, 56-70 (2003).

[80] E. B. van Munster, G. J. Kremers, M. J. W. Adjobo-Hermans, and T. W. J. Gadella, "Fluorescence resonance energy transfer (FRET) measurement by gradual acceptor photobleaching," J. Microsc. 218, 253-262 (2005).

[81] P. I. H. Bastiaens and A. Squire, "Fluorescence lifetime imaging microscopy: spatial resolution of biochemical processes in the cell," Trends Cell Biol. 9, 48-52 (1999).

[82] R. R. Duncan, A. Bergmann, M. A. Cousin, D. K. Apps, and M. J. Shipston, "Multidimensional time-correlated single photon counting (TCSPC) fluorescence lifetime imaging microscopy (FLIM) to detect FRET in cells," J. Microsc. 215, 1-12 (2004).

[83] H. Wallrabe and A. Periasamy, "Imaging protein molecules using FRET and FLIM microscopy," Curr. Opin. Biotechnol. 16, 19-27 (2005).

[84] M. Peter, S. M. Ameer-Beg, M. K. Y. Hughes, M. D. Keppler, S. Prag, M. Marsh, B. Vojnovic, and T. Ng, "Multiphoton-FLIM quantification of the EGFP-mRFP1 FRET pair for localization of membrane receptor-kinase interactions," Biophys. J. 88, 1224-1237 (2005).

[85] T. Kawazoe, K. Kobayashi, and M. Ohtsu, "Optical nanofountain: a biomimetic device that concentrates optical energy in a nanometric area," Appl. Phys. Lett. 86, 103102 (2005).

[86] H. Morawetz, "Studies of synthetic polymers by nonradiative energy transfer," Science 240, 172-176 (1988).

[87] J. P. S. Farinha and J. M. G. Martinho, "Resonance energy transfer in polymer interfaces," Springer Ser. Fluoresc. 4, 215-255 (2008).

[88] G. Srinivas and B. Bagchi, "Detection of collapsed and ordered polymer structures by fluorescence resonance energy transfer in stiff homopolymers: bimodality in the reaction efficiency distribution," J. Chem. Phys. 116, 837-844 (2002).

[89] S. Saini, H. Singh, and B. Bagchi, "Fluorescence resonance energy transfer (FRET) in chemistry and biology: non-Förster distance dependence of the FRET rate," J. Chem. Sci. 118, 23-35 (2006).

[90] R. K. Castellano, S. L. Craig, C. Nuckolls, and J. Rebek, "Detection and mechanistic studies of multicomponent assembly by fluorescence resonance energy transfer," J. Am. Chem. Soc. 122, 7876-7882 (2000). 
[91] C. Cano-Raya, M. D. Fernández-Ramos, and L. F. Capitán-Vallvey, "Fluorescence resonance energy transfer disposable sensor for copper(II)," Anal. Chim. Acta 555, 299-307 (2006).

[92] A. E. Albers, V. S. Okreglak, and C. J. Chang, "A FRET-based approach to ratiometric fluorescence detection of hydrogen peroxide," J. Am. Chem. Soc. 128, 9640-9641 (2006).

[93] H. Zhang and D. M. Rudkevich, "A FRET approach to phosgene detection," Chem. Commun. 1238-1239 (2007).

[94] R. E. Blankenship, Molecular Mechanisms of Photosynthesis (Blackwell Science, Oxford, 2002).

[95] G. D. Scholes, G. R. Fleming, A. Olaya-Castro, and R. van Grondelle, "Lessons from nature about solar light harvesting," Nat. Chem. 3, 763-774 (2011).

[96] G. D. Scholes and G. R. Fleming, "On the mechanism of light-harvesting in photosynthetic purple bacteria: B800 to B850 energy transfer," J. Phys. Chem. B 104, 1854-1868 (2000).

[97] R. J. Cogdell, A. Gall, and J. Köhler, "The architecture and function of the lightharvesting apparatus of purple bacteria: from single molecules to in vivo membranes," Q. Rev. Biophys. 39, 227-324 (2006).

[98] J. Strümpfer, M. Sener, and K, Schulten, "How quantum coherence assists photosynthetic light harvesting," J. Phys. Chem. Lett. 4, 536-542 (2012).

[99] G. D. Scholes, X. J. Jordanides, and G. R. Fleming, "Adapting the Förster theory of energy transfer for modeling dynamics in aggregated molecular assemblies," J. Phys. Chem. B 105, 1640-1651 (2001).

[100] S. Jang and Y.-C. Cheng, "Resonance energy flow dynamics of coherently delocalized excitons in biological and macromolecular systems: recent theoretical advances and open issues," WIREs Comput. Mol. Sci. 2 (in press).

[101] J. L. Herek, W. Wohlleben, R. J. Cogdell, D. Zeidler, and M. Motzkus, "Quantum control of energy flow in light harvesting," Nature 417, 533-535 (2002).

Au: Please update Ref. 100.

[102] F. Kulzer and M. Orrit, "Single molecule optics," Annu. Rev. Phys. Chem. 55, 585-611 (2004). 


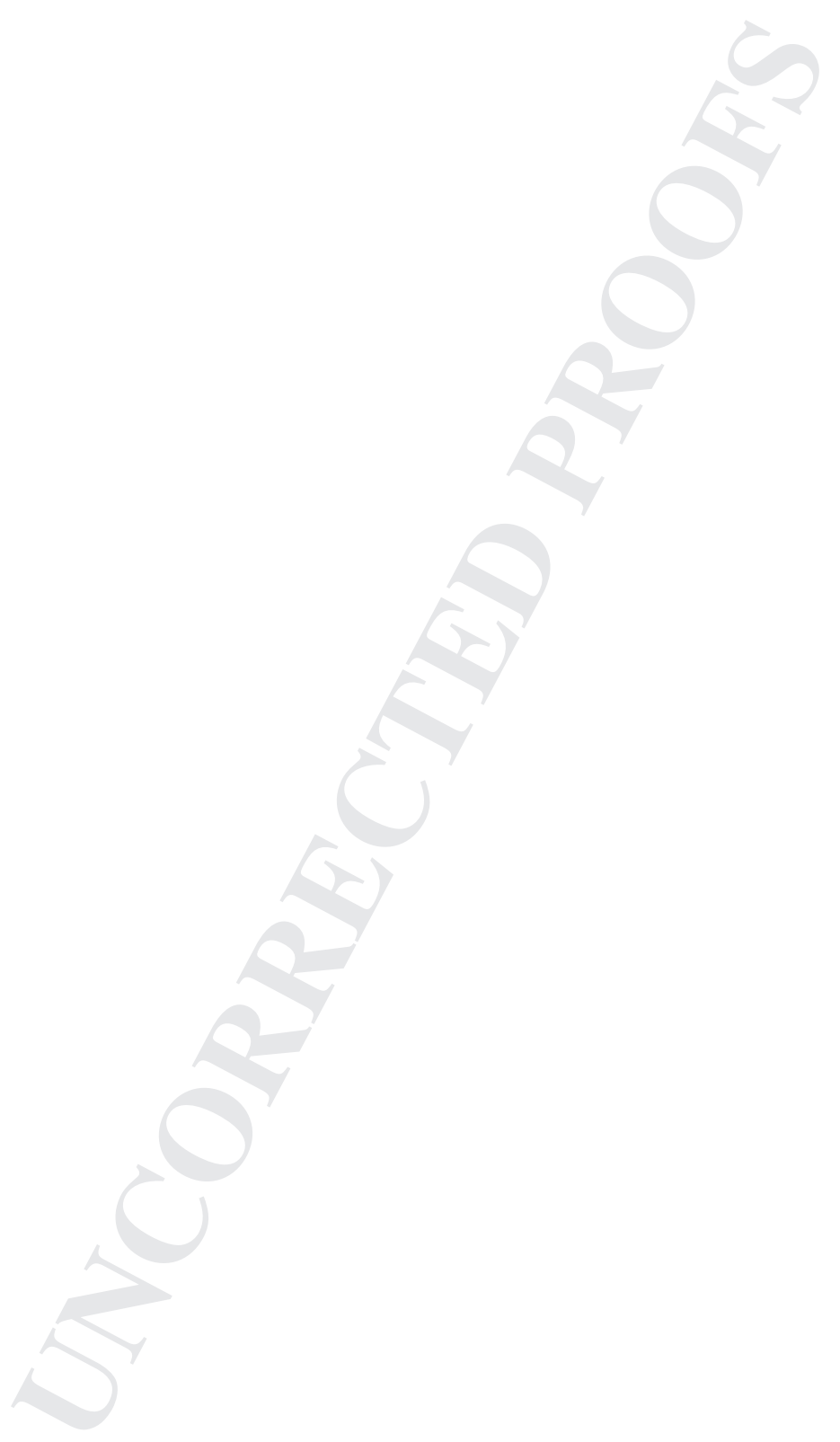

